\title{
Do Healthy People Worry? Modern Health Worries, Subjective Health Complaints, Perceived Health, and Health Care Utilization
}

\author{
Kelly B. Filipkowski • Joshua M. Smyth • \\ Abraham M. Rutchick • Alecia M. Santuzzi • \\ Meera Adya • Keith J. Petrie • Ad A. Kaptein
}

(C) International Society of Behavioral Medicine 2009

\begin{abstract}
Background Modern health worries (MHW) are concerns related to modern or technological features of daily life (e.g., air pollution, x-rays, food additives, etc.), and have been associated with subjective health complaints (SHC) and health care use.

Purpose The MHW scale was expected to predict aspects of health status in healthy individuals (e.g., health care visits, health perceptions, and medication use). SHC was thought to mediate the relationship between MHW and health care use. Likewise, negative affect was considered to mediate the relationship between MHW and SHC.

Method University students $(n=432)$ completed assessments for MHW, SHC, perceptions of health, medication use, and health care visits.

Results MHW were positively related to the number of subjective health complaints with negative affect partially driving this relationship. MHW were negatively related to reports of present health and medication use. MHW were
\end{abstract}

K. B. Filipkowski · J. M. Smyth · A. M. Rutchick •

A. M. Santuzzi $\cdot$ M. Adya

Syracuse University,

Syracuse, NY, USA

K. J. Petrie

University of Auckland,

Auckland, New Zealand

\author{
A. A. Kaptein \\ Leiden University Medical Centre, \\ Leiden, The Netherlands \\ J. M. Smyth $(\bowtie)$ \\ Department of Psychology, Syracuse University, \\ 430 Huntington Hall, \\ Syracuse, NY 13244, USA \\ e-mail: jmsmyth@syr.edu
}

marginally related, whereas SHC were significantly associated with health care utilization.

Conclusion Concerns over modern technology appear to influence symptom reporting, perceptions of current health, medication use, and, to a degree, visits to health care providers even in young healthy samples.

Keywords Modern health worries · Subjective health complaints $\cdot$ Health care use $\cdot$ Health care perceptions

\section{Introduction}

Health care spending and utilization is growing, particularly in the USA $[1,2]$. Paradoxically, this occurs despite overall improvements in objective health and overall lifespan [3]. In recent years, this increase in health care use has been coupled with a rise in "unexplained" symptoms for which there are no objective explanations [4]. Many of these symptoms appear unrelated to organic causes (i.e., disease), but have psychosomatic origins, and are typically defined as subjective health complaints $[5$, 6]. Unfortunately, patients presenting medically unexplained ailments tend to inaccurately recall medical explanations for their symptoms [7]. Those relying on medical remedies are short-changed when doctors lack the ability to adequately treat such complaints. This is problematic as it potentially stigmatizes patients as "over-utilizers" of health care, or worse, resistant to reassurance [8]. The perception of heightened vulnerability that is associated with aspects of modern living has been suggested as one pathway by which individuals interpret such unexplained symptoms [9, 10]. Thus, the current study investigates such concerns as they may drive symptom perception and health care use. 


\section{Modern Health Worries}

Modern health worries (MHW) are defined as the concerns individuals have regarding the health consequences of modern living (e.g., air pollution, X-rays, additives in food, chemicals in household products, etc. [11]). Specifically, modern health concerns may drive the perception of routine daily symptoms as physiological consequences of environmental factors. Furthermore, this has been suggested as being exacerbated by the media's overemphasis of high-risk and disease-related stories [12, 13]. Such focus increases the salience of relatively rare events and can lead to the overestimation of its actual occurrence [14]. Thus, people seeking explanations for perceived health complaints may make a connection between these modern hazards and their symptoms [10, 15].

Modern health concerns have been related to symptom complaints as well as the use of both traditional [11] and alternative health care services [16]. Furthermore, subjective health complaints were found to mediate this relationship [17]. That is, people interpret subjective sensations as signs of poor health resulting from modern influences [10], which drives their attempts to remedy them via conventional and unorthodox means. Overall, evidence supports the suggestion that these concerns serve as a catalyst by which some interpret their complaints as illness and subsequently seek out health care services. It could be further suggested that subjective perceptions of ailment may also impact one's overall perception of health (e.g., if one perceives an ailment, she/he is more likely to report that is indicative of compromised health, at least temporarily). Furthermore, such complaints may similarly drive the use of medication (e.g., as a first measure prior to seeking more professional treatment).

The current study also examined the potential mediating role of negative affect in the relationship between modern health worries and subjective health complaints. The influence of negative affect on the perception and report of symptoms is well-established [18]. Prior research found associations between modern health worries and negative affect [11] as well as subjective health complaints and negative affect [17]; thus, previous studies have controlled for negative affect when examining modern health worries, symptom complaints, and health care utilization. However, in order to assess the magnitude in which negative affect accounts for this relationship, we examined its potential mediating role between modern health concerns and symptom reports.

Prior research has relied on individuals related to the health care profession and those taking part in national surveys of health status and health service utilization (e.g., patient care). It could be suggested that increased familiarity with health care may skew one's perception of illness (e.g., "medical student syndrome," salience effects, etc.), and modern health worries would be less relevant to health care utilization of healthy individuals. Thus, it remains important to assess whether the predictability of modern health concerns are related to general medically-related processes among those not intimately connected to health services (broadly defined).

\section{Study Objectives and Predictions}

The present study investigates the relationship of modern health worries (as assessed by the validated MHW scale) to various health-related outcomes of young, healthy individuals. Based on associations between modern health worries, subjective health complaints, and health care utilization in previous studies, we predict:

1. Modern health worries will be related to visits to a health care provider, perceptions of current health, and medication use.

2. Subjective health complaints will mediate the relationships between modern health worries and each of the aforementioned health outcomes (e.g., health care visits, perceptions of health, and medication use).

3. Negative affect is expected to mediate the relationship between modern health worries and subjective health complaints.

\section{Method}

Participants

A total of 432 students from a psychology course enrolled in the study using an online (internet) registration system. In order to preserve anonymity in responding, demographic information was collected in a manner impossible to link with specific participant responses (unfortunately precluding analysis of individual differences as predictors). The sample was $60 \%$ female with an age range of 19 to 38 years $(\mathrm{M}=19.40$ years; $\mathrm{SD}=1.43$ years). Race/ethnicity was reported as $77 \%$ Caucasian, 9\% Asian, 5\% AfricanAmerican, 4\% Latino, and less than 3\% identified themselves as "other" ( $\sim 2 \%$ declined to report their ethnicity).

Procedure and Instruments

Upon completing informed consent, participants anonymously completed a packet of measures (described later). All participants received research participation credit as partial fulfillment of psychology course requirements. 
Modern Health Worries Scale [11] Twenty-five items assess concerns regarding the effects that modern living may have on health. Although modern health worries may fluctuate with contextual factors, they are conceptualized as reflecting more general trait-like concerns over aspects of modern living $[10,11] .{ }^{1}$ It consists of a total score and can also generate four subscales (toxic interventions, environmental pollution, tainted food, and radiation). Responses range from 1 (no concern) to 5 (extreme concern) with higher total scores indicating greater concern of modern technology's influence on health outcomes (sample $\mathrm{M}=62.95 ; \mathrm{SD}=18.21$ ). Cronbach's alpha for the total scale was 0.94 . We utilized the total composite score in analyses to control type-I error.

Subjective Health Complaints (SHC; [19]) This 28-item scale measures reported symptoms. Participants rated the extent to which they had been affected by a particular symptom in the past month, ranging from 0 (not at all) to 3 (serious). This scale produces a total subjective health score and can generate five subscales (pseudoneurology complaints, flu symptoms, allergy symptoms, gastrointestinal problems, and musculoskeletal pain). Cronbach's alpha for the total scale was an acceptable 0.81 ; the total scale mean was $16.99(\mathrm{SD}=9.06)$.

Positive and Negative Affect [20] Participants indicated on 20 items the extent to which they felt, on average, a number of specific emotions, ranging from 1 (very slightly or not at all) to 5 (extremely). The current study focused on the ten items comprising the negative affect subscale. Higher summed scores indicate higher levels of negative affect $(\mathrm{M}=19.89 ; \mathrm{SD}=5.96)$; Cronbach's alpha for this subscale was 0.83 .

Use of Health Care Services Participants indicated how often they have visited various types of health care providers in the past 12 months. These responses were summed to provide a total number of health care provider visits for each participant $(\mathrm{M}=5.74 ; \mathrm{SD}=8.66)$.

Current Health Perceptions Participants provided a global assessment of their current health, ranging from 1 (terrible) to 7 (excellent; $\mathrm{M}=5.33 ; \mathrm{SD}=0.95$ ). Single-item measures of global health have been found to robustly and reliably predict health and mortality outcomes [21].

Medication Use Students reported type of medication use (i.e., prescribed, over-the-counter, or homeopathic) in the

\footnotetext{
${ }^{1}$ Due to the relatively new development of the MHW scale, there are no longitudinal studies that confirm this conceptualization. This is discussed as a limitation and suggestion for future research.
}

past 12 months. The number of responses were summed across all three categories to provide a total medication use score $(\mathrm{M}=2.42 ; \mathrm{SD}=1.78)$.

\section{Analytic Strategy}

Our primary goal was to examine the relationship between modern health worries and health-related outcomes. In general, analyses consisted of regressions using the total MHW score to predict, separately, total number of visits to health care providers, perceptions of current health, and use of medication. Visits to health care providers and use of medications showed positively skewed response distributions. Thus, these responses were analyzed using Poisson regression models, ${ }^{2}$ which tend to assume a more suitable distribution for positively-skewed count responses [22]. Subjective health complaints were expected to mediate the relationships between modern health worries and each of the three health-related outcome measures. In accord with mediation requirements (e.g., [23-25]), distinct regression analyses were conducted to determine if: (a) MHW significantly predicts SHC; (b) MHW predicts health care use, health care perceptions, and medication use; (c) SHC significantly predict health care use, health care perceptions, and medication use; and (d) the relationship between MHW and any of the three health-related outcomes decreases or becomes nonsignificant when controlling for SHC. Similarly (utilizing the same mediation criteria), separate regression analyses were conducted to examine the influence of negative affect on the relationship between modern health worries and subjective health complaints.

\section{Results}

\section{Modern Health Worries ${ }^{3}$ and Health Outcomes}

Poisson regression analyses indicated that modern health worries were marginally related to the number of health care visits $(b=.002, \mathrm{SE}=.001, p=.072)$. In addition, participants' modern health worries were significantly related to their report of present health $(b=-.005$, $\mathrm{SE}=.003, p=.049)$ such that more worries were associated with poorer perceptions of health status. Modern health worries were negatively related to the frequency of

\footnotetext{
$\overline{{ }^{2} \text { These models }}$ used a logarithmic link function; thus, regression weights should be interpreted as logarithmic transformations of the weights.

${ }^{3}$ Prior to conducting regression analyses, a factor analysis of the MHW scale was conducted. We found the same general structure that previous research has validated and generalized [11, 17]. Further information is available upon request from the authors.
} 
medication use $(b=-.005, \mathrm{SE}=.002, p=.005)$. Thus, more concerns over modernity coincided with more visits to a health care provider but less consumption of medication (please refer to Fig. 1 for a detailed summary of all path coefficients within the hypothesized model).

\section{Mediator Role of Subjective Health Complaints}

Modern health worries were significantly associated with the total subjective health complaints score $(b=.11$, $\mathrm{SE}=.03, p<.001)$; thus, in general, participants with increased worries also reported more health complaints. Subjective health complaints were expected to serve a mediating role between modern health worries and each of the three main dependent variables (i.e., health care visits, health perceptions, and medication use). Below, the association between subjective health complaints and each health outcome is provided in its respective section. In addition, for each outcome, the absolute change between the original path coefficients and the new coefficients (i.e., with the inclusion of SHC in the model) is indicated by $\Delta b$. Both the original and new pathway coefficients for each outcome can be located on Fig. 1.

Health Care Utilization There was a statistical trend between health care visits and total modern health worries $(p<.08)$. This was deemed sufficient criteria to conduct mediation analysis as a significant pathway between MHWs and health care visits is considered unnecessary [24, 25]. In addition, subjective health complaints co-occurred with reported estimates of health care provider visits $(b=.02, \mathrm{SE}=.002, p<.001)$. Furthermore, when SHC were added to the prediction of health provider visits, the relationship between modern health worries and number of visits to health care providers substantially decreased $(\Delta b=.003 ;$ Deviance $=2209.51$, $d f=373$; see Fig. 1). The mediated effect was demonstrated to be significant, $z=3.44, p<.001$ [26] providing evidence that subjective health complaints largely drive the relationship between modern health worries and health care utilization.

Health Perceptions Participants' perception of their health at the present time was associated with their overall reports of subjective health complaints $(b=-.03, \mathrm{SE}=.005$, $p<.001)$. Thus, fewer overall reported subjective health complaints coincided with better overall perceptions of present health status. When subjective health complaints were included in the model, the relationship between modern health worries and health perceptions became nonsignificant $\left(\Delta b=.003 ; \Delta R^{2}=.10\right.$; see Fig. 1). A Sobel test [26] verified that this was a significant indirect effect, $z=-3.13, p<.001$. Thus, the relationship between modern health worries and health perceptions is explained, in part, by increased complaints of symptoms.

Medication Use The multiple regression model suggested that both modern health worries $(b=-.008, \mathrm{SE}=.002$, $p<.001)$ and subjective health complaints $(b=.02, \mathrm{SE}=.004$, $p<.001)$ each served as unique predictors of medication use (modern health worries, $\Delta b=.003$; Deviance $=456.62, d f=$ 373; see Fig. 1). Thus, the inclusion of subjective health complaints did not appear to mediate the relationship between modern health worries and medicine use.

\section{Negative Affect}

In the current study, modern health worries $(b=.09$, $\mathrm{SE}=.02, p<.001)$ and subjective health complaints
Fig. 1 Modern health worries and health-related outcomes: path coefficients of the original and mediated relationships

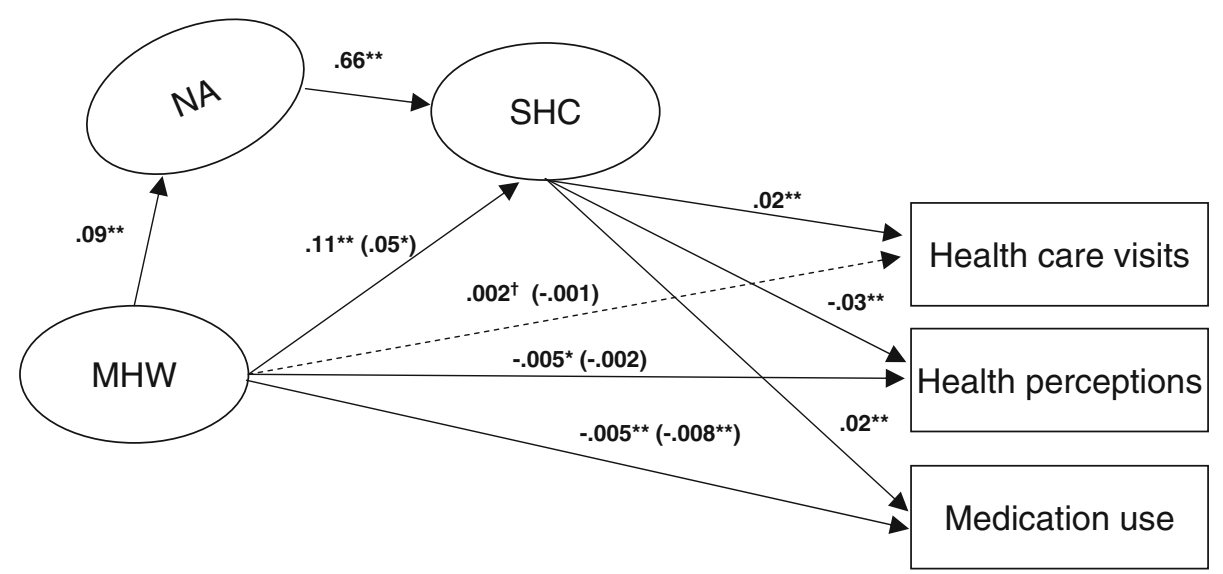

${ }^{\dagger} p<.08 ;{ }^{*} p<.05,{ }^{* *} p<.01$

Weights are unstandardized regression coefficients

Weights in () = new weights after mediation (indirect effects)

Lines represent original non-mediated effects 
$(b=.66, \mathrm{SE}=.07, p<.001)$ were significantly related to negative affect. Modern health worries significantly predicted subjective health complaints $(b=.11, \mathrm{SE}=.03$, $p<.001$ ); when negative affect was included in the model, this effect was reduced, although modern health worries still remained a significant predictor $\left(\Delta b=.06, \Delta R^{2}=.17\right.$; see Fig. 1). A Sobel test [26] indicated that negative affect significantly mediated the influence of modern health worries on subjective health complaints $(z=4.10, p<.001)$.

\section{Discussion}

Prior studies have demonstrated a connection between concerns over modern living and the utilization of health care $[11,16]$, with subjective health complaints the apparent mediator [17]. Our study provides further evidence of the stability and generalizability of the MHW scale. That is, young, healthy university students responded similarly to previous participants, indicating that modern health concerns are not unique to those familiar with medicine. Specifically, those who reported more modern health worries tended to complain of more symptoms and report poorer health. In this study, subjective health complaints were also associated with perceptions of current health and were found to mediate the relationship between modern health worries and perceptions of health. This suggests that concern over various aspects of modern day living may influence reported health by impacting the appraisal of experienced symptoms $[9,17]$. This is further supported by the mediating influence of negative affect on subjective health complaints (discussed in detail later).

In addition, subjective health complaints were significantly related to health care visits, indicating that these symptoms may influence our health care habits. The relationship between modern health worries and the use of health care services was (statistically) of marginal significance, not providing strong support for the association between modern health worries and health care use. The students in our study were, in general, quite healthy, reducing statistical power (via restricted range and variance) to detect an association between modern health worries and health care use. Our sample also may not have been as familiar with the medical field (and use of health care) as prior samples (that often included medically trained personnel). This adds support, however, to the idea that modern health worries may predispose an individual to perceive daily sensations in a way that promotes health care utilization.

Regarding the use of medication, modern health worries predicted less medication use, whereas subjective health complaints were related to increased consumption. This was unexpected as modern health worries were considered to drive health care use, such as medication (e.g., more concern would translate into increased consumption of medication). One interpretation is that individuals reporting more worries may consider over-the-counter medicines as insufficient in alleviating their ailments and thus utilize more extensive health care services. Similarly, it could also be suggested that individuals who express more concern regarding modernity also view medications skeptically, perhaps even considering them potential hazards to their health $[27,28]$. These post hoc explanations should be taken cautiously as this was not directly tested and is speculative. It does indicate, however, a need to clarify the mechanism by which modernity concerns reduce medication use. A difference may exist between those who avoid medicine based on fear versus those who are skeptics of its effectiveness. Diverse intervention and/or educational strategies may be required to either reduce fears or unnecessary reliance on an overburdened health care system. Future research should examine the specific reason(s) for a reduction in medication use associated with modern health concerns.

Additionally, as negative affect is recognized to influence symptom reports, we assessed the influence of general negative mood. Prior studies have statistically controlled for negative affect $[11,17]$ so as to account for differences in general mood dispositions. This allows for more precise prediction of subjective health complaints and health outcomes; however, this does not specifically demonstrate the process by which negative affect may influence the relationships between modern health worries and healthrelated outcomes. We demonstrated that negative affect partially mediates the relationship between modern health worries and subjective health complaints. That is, modern health worries lead to subjective health complaints in part, but not fully, by increasing negative affect. This would be consistent with the "symptom perception hypothesis" in which people with higher negative affect are more likely to attend to bodily sensations, interpret them as indicative of disease, and explicitly express such concerns [29]. Thus, perhaps modern health worries generate an overall negative schema by which physical sensations are perceived and appraised. Yet, the relationship between modernity concerns and symptom reports was only partially mediated by general negative mood, suggesting that there are other factors that add to the interpretation of symptoms. It could be argued that stress may influence this relationship as it has been shown to influence one's perception of sensations as indicating disease as well as increasing the likelihood to seek health care [30]. In addition, prior experience, expectations, and the seriousness of the symptoms can all exert influence on symptom appraisal [31-33]. For instance, those who have repeatedly dealt with a medical condition tend to rate it as less threatening than those who have no 
history of it [31]. Therefore, in addition to subjective health complaints and general negativity, there are other plausible mediating variables that require further examination.

\section{Limitations and Future Directions}

The self-report questionnaires used in this study required participants to retrospectively recall their physical and mental well-being. Self reported use of psychological and physical health services has demonstrated moderate reliability at 3 months [34]; however, recall for health care utilization in the current study covered a relatively lengthy span of 12 months, which may result in errors. In contrast, health-related visits are likely relatively salient and may be less subject to recall limitations. Nonetheless, future research might consider utilizing more objectively measured health outcomes and administrative records of health care use to decrease possible reporting biases.

The current study's use of a concurrent assessment of these relationships limits clear causal interpretation. These issues should continue to be studied and extended to include prospective and experimentally based paradigms. Likewise, a longitudinal study is needed to confirm the conceptualization of modern health worries as a trait-like variable.

This study (and most previous research) has used relatively young participants. Recently, a study utilizing a more diverse age range found that older individuals reported less concern regarding modernity [16]. Thus, some worries regarding consequences of modern living may be more relevant or salient to younger individuals. Future research should focus on the inclusion of older individuals to assess this potential discrepancy across age cohorts. Further generalizability of the MHW scale was established by utilizing a sample with more diverse characteristics than previous research (e.g., healthy, young individuals with no connection to the health profession). However, the cultures within which the MHW scale has demonstrated consistent findings are relatively similar to one another. Several studies have suggested that some cultures do not interpret health within the same contextual framework as most westernized cultures $[35,36]$. Inclusion of individuals from diverse cultures that may interpret and respond to symptoms differently would be informative.

\section{Conclusions}

These data provide additional evidence for the structure and function of the MHW scale across different sample characteristics. Specifically, concerns regarding modernity influence individuals' views of physical health and health care behaviors. Individuals reporting more modern health worries tended to complain of more symptoms, report poorer health, yet consume less medication. Subjective health complaints were associated with perception of current health and largely mediated the relationship between modern health worries and perceptions of health. Modern health worries and subjective health complaints were both related to negative affect, and negative affect partially mediated the relationship between them. As such, physicians and other health care providers attending to patients presenting with unexplained symptoms may find this scale (or consideration of modern health worries more generally) clinically informative.

Acknowledgments The authors are grateful for the contributions made to this project by Lien Tran.

\section{References}

1. Catlin A, Cowan C, Heffler S, Washington B. National health spending in 2005: the slowdown continues. Health Aff. 2007;26:142-53.

2. Smith C, Cowan C, Sensenig A, Catlin A. Health spending growth slows in 2003. Health Aff. 2005;24:185-94.

3. Barsky AJ. The paradox of health. N Engl J Med. 1988;318:4148 .

4. Eriksen HR, Hellesnes B, Staff P, Ursin H. Are subjective health complaints a result of modern civilization? Int J Behav Med. 2004; $11: 122-5$.

5. Nimnuan C, Hotopf M, Wessely S. Medically unexplained symptoms: how often and why are they missed? Q J Med. 2000;93:21-8.

6. Ursin H. Sensitization, somatization and subjective health complaints. Int J Behav Med. 1997;4:105-16.

7. Rief W, Heitmuller AM, Reisberg K, Ruddel H. Why reassurance fails in patients with unexplained symptoms: an experimental investigation of remembered probabilities. PLoS Med. 2006;3:1266-72.

8. Fink P, Rosendal M. Recent developments in the understanding and management of functional somatic symptoms in primary care. Curr Opin Psychiatry. 2008;21:182-8.

9. Petrie KJ, Booth RJ, Elder H, Cameron LD. Psychological influences on the perception of immune function. Psychol Med. 1999;29:391-7.

10. Petrie KJ, Wessely S. Modern worries, new technology, and medicine: new technologies mean new health complaints. Br Med J. 2002;324:690-1.

11. Petrie KJ, Sivertsen B, Hysing M, Broadbent E, Moss-Morris R, Eriksen HR, et al. Thoroughly modern worries: the relationship of worries about modernity to reported symptoms, health and medical care utilization. J Psychosom Res. 2001;51:395-401.

12. Frost K, Frank E, Maibach E. Relative risk in the news media: a quantification of misrepresentation. Am J Public Health. 1997;87:842-5.

13. Hofmann B. The paradox of health care. Health Care Anal. 2001;9:369-86.

14. Gilovich T, Griffin D, Kahneman D. Heuristics and biases: the psychology of intuitive judgment. New York: Cambridge University; 2002.

15. Petrie KJ, Broadbent EA, Kley N, Psych D, Moss-Morris R, Horne $\mathrm{R}$, et al. Worries about modernity predict symptom 
complaints after environmental pesticide spraying. Psychosom Med. 2005;67:778-82.

16. Furnham A. Are modern health worries, personality and attitudes to science associated with the use of complementary and alternative medicine? Br J Health Psychol. 2007;12:229-43.

17. Kaptein AA, Helder DI, Kleijn WC, Rief W, Moss-Morris R, Petrie KJ. Modern health worries in medical students. J Psychosom Res. 2005;58:453-7.

18. Pennebaker JW, Watson D. The psychology of somatic symptoms. In: Kirmayer LJ, Robbins JM, editors. Current conceptions of somatization: research and clinical perspectives. Washington, DC: American Psychiatric Association; 1991. p. 21-35.

19. Eriksen HR, Ihlebaek C, Ursin H. A scoring system for subjective health complaints. Scand J Public Health. 1999;27:63-72.

20. Watson D, Clark L, Tellegen A. Development and validation of brief measures of positive and negative affect: the PANAS scales. J Pers Soc Psychol. 1988;54:1063-70.

21. Idler EL, Benyamini Y. Self-rated health and mortality: a review of twenty-seven community studies. J Health Soc Behav. 1997;38:21-37.

22. Agresti A. Categorical data analysis. 2nd ed. Hoboken: Wiley; 2002.

23. Baron RM, Kenny DA. The moderator-mediator variable distinction in social psychological research: conceptual, strategic and statistical considerations. J Pers Soc Psychol. 1986;51:1173-82.

24. James LR, Brett JM. Mediators, moderators, and tests for mediation. J Appl Psychol. 1984;69:307-21.

25. MacKinnon DP, Fairchild AJ. Current directions in mediation analysis. Curr Dir Psychol Sci. 2009;18:16-20.

26. Sobel ME. Asymptotic confidence intervals for indirect effects in structural equation models. In: Leinhardt S, editor. Sociological methodology 1982. Washington: American Sociological Association; 1982. p. 290-312.

27. Horne R. Representations of medication and treatment: advances in theory and measurement. In: Petrie KJ, Weinman JA, editors.
Perceptions of health and illness: current research and applications. Amsterdam: Harwood Academic Publishers; 1997. p. 15588.

28. Horne R, Weinman J, Hankins M. The beliefs about medicines questionnaire: the development and evaluation of a new method for assessing the cognitive representation of medication. Psychol Health. 1999;14:1-24.

29. Watson D, Pennebaker JW. Health complaints, stress, and distress: exploring the central role of negative affectivity. Psychol Rev. 1989;96:234-54.

30. Cohen S, Williamson M. Stress and infectious disease in humans. Psychol Bull. 1991;109:5-24.

31. Jemmott JB, Croyle RT, Ditto PH. Commonsense epidemiology: self-based judgments from laypersons and physicians. Health Psychol. 1988;7:55-73.

32. Klebanov PK, Jemmott JB. Effects of expectations and bodily sensations on self-reports of premenstrual symptoms. Psychol Women Q. 1992;16:289-310.

33. Martin R, Leventhal H. Symptom perception and health careseeking behavior. In: Raczynski JM, Leviton LC, editors. Handbook of clinical health psychology: vol. 2. Disorders of behavior and health. Washington: American Psychological Association; 2004. p. 299-328.

34. Chung S, Domino ME, Jackson EW, Morrissey JP. Reliability of self-reported health service use: evidence from the women with co-occurring disorders and violence study. J Behav Health Serv Res. 2008;35:265-78.

35. Brough M, Gorman D, Ramirez E, Westoby P. Young refugees talk about well-being: a qualitative analysis of refugee youth mental health from three states. Aust J Soc Issues. 2003;38:193208.

36. Reiff M, Zakut H, Weingarten MA. Illness and treatment perceptions of Ethiopian immigrants and their doctors in Israel. Am J Public Health. 1999;89:1814-8. 